\title{
Modeling and Simulation of Reconfigurable Intelligent Surfaces for Hybrid Aerial and Ground-based Vehicular Communications
}

\author{
Karsten Heimann \\ Communication Networks Institute \\ TU Dortmund University, Germany \\ karsten.heimann@tu-dortmund.de \\ Manuel Patchou \\ Communication Networks Institute \\ TU Dortmund University, Germany \\ manuel.mbankeu@tu-dortmund.de
}

\begin{abstract}
The requirements of vehicular communications grow with increasing level of automated driving and future applications of intelligent transportation systems (ITS). Beside the ever-increasing need for high capacity radio links, reliability and latency constraints challenge the mobile network supply. While for example the millimeterwave spectrum and $\mathrm{THz}$-bands offer a vast amount of radio resources, their applicability is limited due to delicate radio channel conditions and signal propagation characteristics. Reconfigurable intelligent surfaces (RISs) as part of smart radio environments (SREs) of future ITS infrastructure promise improved radio link qualities by means of purposeful cultivation of passive reflections. With this, obstructed mmWave or THz beams can be guided around obstacles through RIS reflection paths to improve the otherwise limited coverage. In this article, application use cases of RIS-enhanced vehicular communications are proposed. Beside static deployments of RISs at exterior walls of buildings, unmanned aerial vehicles (UAV) could provide reflection capabilities on demand, while future vehicles could - in a visionary approach - consist of meta-material allowing for their opportunistic utilization within an enriched SRE. Results of a case study based on our multi-scale mobility and network simulation model clearly highlight the potential of RIS deployment for hybrid vehicular communication scenarios. Path loss and outage percentages can be reduced considerably.
\end{abstract}

\section{CCS CONCEPTS}

- Networks $\rightarrow$ Physical links; Mobile networks; Network performance analysis; Network simulations; Wireless access points, base stations and infrastructure; • Computing methodologies $\rightarrow$ Simulation environments; Discrete-event simulation;

Permission to make digital or hard copies of all or part of this work for personal or classroom use is granted without fee provided that copies are not made or distributed for profit or commercial advantage and that copies bear this notice and the full citation on the first page. Copyrights for components of this work owned by others than the author(s) must be honored. Abstracting with credit is permitted. To copy otherwise, or republish, to post on servers or to redistribute to lists, requires prior specific permission and/or a fee. Request permissions from permissions@acm.org.

MSWiM '21, November 22-26, 2021, Alicante, Spain

(C) 2021 Copyright held by the owner/author(s). Publication rights licensed to ACM

ACM ISBN 978-1-4503-9077-4/21/11 ..\$15.00

https://doi.org/10.1145/3479239.3485700

\author{
Benjamin Sliwa \\ Communication Networks Institute \\ TU Dortmund University, Germany \\ benjamin.sliwa@tu-dortmund.de \\ Christian Wietfeld \\ Communication Networks Institute \\ TU Dortmund University, Germany \\ christian.wietfeld@tu-dortmund.de
}

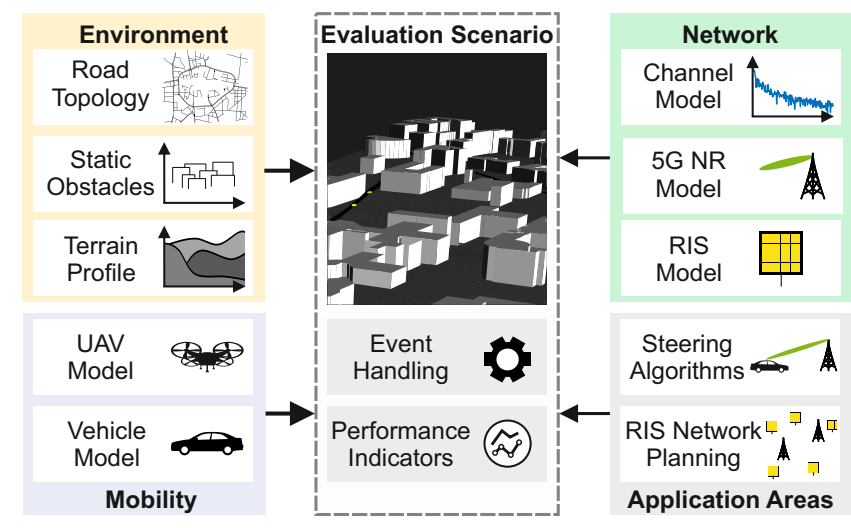

Figure 1: The overall system architecture model of the proposed simulation framework for the evaluation of reconfigurable intelligent surface (RIS)-enhanced hybrid aerial and ground-based vehicular communications.

\section{KEYWORDS}

Smart radio environments; millimeter wave; reconfigurable intelligent surfaces; vehicular communications

\section{ACM Reference Format:}

Karsten Heimann, Benjamin Sliwa, Manuel Patchou, and Christian Wietfeld. 2021. Modeling and Simulation of Reconfigurable Intelligent Surfaces for Hybrid Aerial and Ground-based Vehicular Communications. In Proceedings of the 24th ACM International Conference on Modeling, Analysis and Simulation of Wireless and Mobile Systems (MSWiM '21), November 22-26, 2021, Alicante, Spain. ACM, New York, NY, USA, 8 pages. https: //doi.org/10.1145/3479239.3485700

\section{INTRODUCTION}

RISs show disruptive potential in enabling beyond line-of-sight (LOS) communications in vehicular networks for technologies having a critical dependency on unobstructed LOS transmissions [4, 17]. The great data requirements issued by novel applications such as autonomous and connected driving have motivated the exploration of the millimeter-wave (mmWave) spectrum and $\mathrm{THz}$ bands in search of higher bandwidths. Network communications in these bands however suffer greatly from obstacle-induced path loss, hereby posing an additional challenge to their utilization in urban environments. 
The RIS concept has been presented as a promising solution for beyond LOS coverage in mmWave vehicular networks in [5]. The general potential for coverage enhancement was illustrated through the simulative investigation of a vehicular application use-case using our lightweight ICT-centric mobility simulation (LIMoSim) from [14], a mobility and network co-simulation model framework with particular support for hybrid aerial and ground-based vehicular networks. Based on these preparatory works, Figure 1 depicts the proposed system architecture model. It composes capabilities for environment definitions, various mobility models and an integrated network simulator based on ns-3 and its recent extension $5 G-L E N A$ for $5 G$ networks from [13], which has been extended by a RIS channel model as elaborated in more detail in the further course.

Beside the contribution of a system-level simulation framework, this work also presents novel solution approaches offered by the integration of the RIS technology into future vehicular networks based on Figure 2. While the strategic deployment of RIS in urban environments can enhance network coverage and facilitate vehicleto-vehicle $(\mathrm{V} 2 \mathrm{~V})$ communications, a more flexible exploitation of the RIS potential lies in extending their mobility. By embedding RISs on unmanned aerial vehicles (UAVs), which offer higher degrees of freedom regarding position and trajectory, a more flexible and responsive network provisioning can be realized. Reacting more timely to sudden situations or events such as accidents and traffic jams would also become possible. In addition, ubiquitous RIS deployments in a smart radio environment (SRE) would allow for a controlled but opportunistic utilization of RIS reflected communication paths. It is assumed opportunistic, since in contrast to the operation of a UAV dedicated to offer a RIS reflection occasion, most of the vehicles may follow their objectives to deliver goods or person and thus their mounted RISs are only available by chance.

The contributions provided by this work are as follows:

- Investigation of the potential of intelligent surfaces and SREs for hybrid vehicular communications at mmWave and $\mathrm{THz}$ bands.

- Presentation of realistic application use cases in the context of future intelligent transportation system (ITS).

- Development of a RIS enhanced system architecture model.

- Example evaluation of case studies by means of joint mobility and network simulations.

The remainder of this work is structured as follows: Referring to related work, insights are given on the fundamentals of intelligent surfaces and their prospective advantages for future mobile networks as well as the integration of UAVs bolstering ITS. Section 3 elaborates on the main concept of RISs-aided hybrid vehicular communication networks and gives an outlook towards a ubiquitous deployment and opportunistic utilization by also considering open challenges. The following section 4 illustrates the proposed simulation framework and contains the conducted simulation studies highlighting the envisaged potentials. Finally, a summary of the key findings concludes this work.

\section{RELATED WORK}

In [3], the authors provide an extensive literature survey on recent and future technology trends for next generation mobile networks.
Among the various prospective use cases mentioned in this article, a smart infrastructure is believed to enable comprehensive network coverage. Thus, it facilitates the omnipresence of wireless systems with the aid of controllable wireless signal propagation. In addition, applications like multi-sensory holographic teleportation, autonomous cyber-physical systems, and intelligent industrial automation put high requirements on the performance of subsequent mobile networks. To meet these challenges, smart radio environments enabled by the RIS technology are envisioned as one of the key drivers for $6 \mathrm{G}$ and beyond. Especially wireless communication at the mmWave and $\mathrm{THz}$ bands, which suffer from limited distances and sparse coverage, can be improved by controlled reflections. The RISs are particularly suitable, because their reflection gain strongly depends on the dimension in relation to the wavelength of the radio signal. Consequently, shorter wavelengths as in case of these bands allow for a higher gain per RIS area and thus a more efficient surface size utilization or in turn, a smaller required surface size compared to the conventional sub $6 \mathrm{GHz}$ bands for example.

The RIS technology derives its origin from the intelligent metasurfaces with its development and state of the art especially in terms of realization concepts and material physics considerations thoroughly elaborated in [15].

An overview of the RIS technology - also called intelligent reflecting surface (IRS) - and its applications in wireless networks is provided in [17]: With RIS-controlled reflections, signal propagation can be guided around obstacles leading to a virtually extended LOS path. In doing so, the main application of RIS can be regarded as providing a more comprehensive network coverage by circumventing blockages. However, the physical layer security profits from controlled destructive signal superposition at eavesdroppers as well. Concerning the controlled superposition of direct and reflected signals at the receiving node, also the rank of multiple input multiple output (MIMO) channels can be increased or the interference by other base stations can be mitigated at the cell edge. For these applications, the deployment of RISs at proper locations is a crucial task to fully leverage the potential of RIS-enhanced mobile networks, which might be supported by machine learning-based techniques.

The article [6] gives an overview of holographic MIMO surfaces, with RIS as one manifestation. In doing so, different technology approaches and concepts like discrete surfaces as passive reflectors are categorized. Generally, there are four functionalities of passive reflectors defined allowing for the control of wireless signal propagation: Polarization, scattering, focusing, and absorption. Besides an improved radio link quality especially for outdoor-to-indoor applications, the surfaces even bear the potential of accurate indoor positioning due to their spatial resolution.

In addition to [5], authors in [4] have analyzed the potentials of RIS-enabled communications in challenging vehicular environments. For providing coverage in so-called dark zone areas, which are affected by signal blocking, the authors utilize deep reinforcement learning for joint resource scheduling and passive beamforming.

In ITS, UAVs are expected to become a key enabler for fully automated transportation according to [9]. Beside parcel delivery tasks for which they can provide a performance boost while complying with complex constraints such as the sanitary measures of the COVID-19 pandemic as proposed in [12], they can act as report 
agents supporting accident ambulance by providing information of crash sites and establishing communication links to involved persons. In addition, UAVs can act as communication relay and road side units (RSUs) to offer better radio conditions for vehicleto-everything (V2X) communications.

Furthermore, the detailed tutorial [18] emphasizes the advantageous opportunities of UAV-assisted cellular networks. While the remote control of UAVs connected via cellular networks has virtually no range limitation, applications like flying relays profit from a high LOS probability to both base stations and user/terminal devices. In addition to global navigation satellite system (GNSS), the cellular-aided localization may even enhance the robustness and performance of UAV navigation. Although the authors in [18] do not take SREs and RISs into account, sophisticated models for the air-to-air and air-to-ground communication performance are presented. The UAV-aided cellular coverage and communication link performance might be even further improved by means of SREs as studied in the subsequent sections.

As pointed out by a visionary analysis in [2], RIS-mounted UAV systems offer the potential of exploiting the unique mobility characteristics of these aerial vehicles for highly efficient on-demand network provisioning. An optimization approach based on machine learning regarding the location and configuration of such a flying RIS is proposed in [19].

While the emerging topic of the RIS technology and its application in vehicular networks is mostly being addressed by means of analytical and numerical simulations in literature, this work focuses on a system level, discrete event simulation.

\section{RIS-ENHANCED HYBRID VEHICULAR COMMUNICATIONS}

As discussed earlier, the controlled utilization of additional reflected paths may not only enhance the multipath richness or rank of MIMO channels, but also extend the network coverage in terms of an improved accessibility of non-line-of-sight (NLOS) regions. This especially applies for wireless communications at the mmWave and $\mathrm{THz}$ bands, where shorter wavelengths allow for reduced size requirements of RIS installations. However, vehicular communications comes with various challenges, which could be met by utilizing the RIS technology as indicated in Figure 2. The utilization of mmWave and $\mathrm{THz}$ bands is heavily based on directional antennas, primarily with an electrically steerable main lobe (e.g. through a phased array). Due to the directional propagation of these signals, the beams need to track mobile users. For this reason, the beam management is a crucial challenge of current $5 \mathrm{G}$ networks operating in the so-called frequency range 2 (FR2), which is the mmWave domain.

With RISs, the available coverage area is extended, while beam management methods may still be applicable to the reflection beams. This allows for persisting with mobile network supply even during LOS blockages. Nevertheless, the vehicular mobility still involves more complexity than the so-called fixed wireless access (FWA) like for broadband provisioning of stationary network subscribers. For a proper alignment of the reflection direction, a precise estimation of the channel state information (CSI) may be required and could be supported geometry-based computations utilizing location and

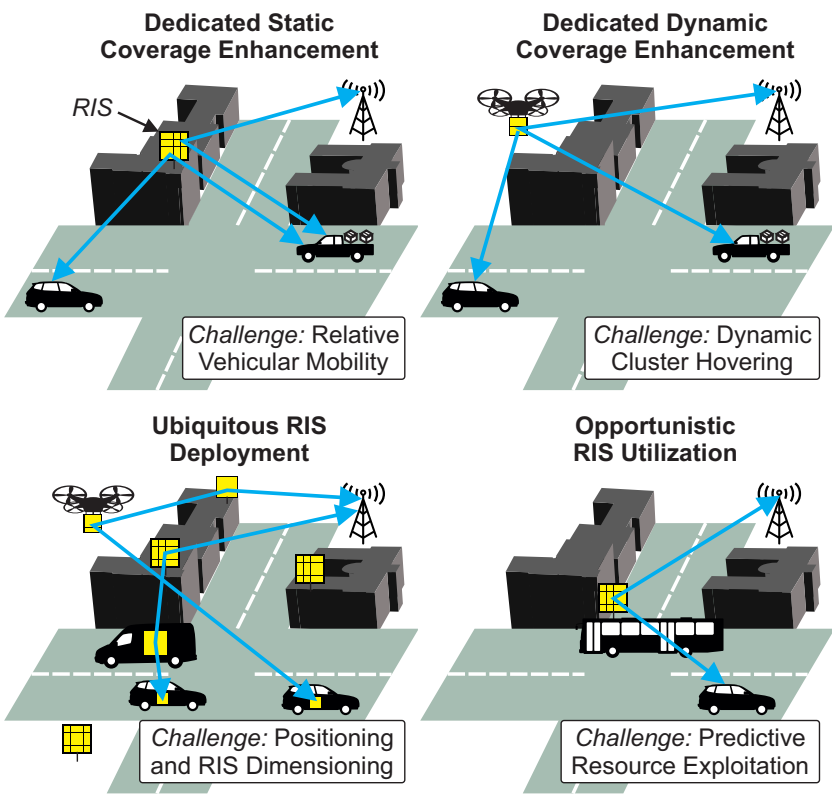

Figure 2: Different RIS integration options within hybrid vehicular networks. The application of RISs is believed to escalate the performance of vehicular communications not only in case of static deployments but also when mounted on UAVs. In addition to a (static or dynamic) placement exclusively dedicated to improve mobile networks, a ubiquitous RIS deployment may be utilized opportunistically in future.

map information. In spite of that, also the volatile V2V links may benefit from the reliable and predictable disposability of static RIS installations dedicated to the purpose of enhanced coverage or network availability.

In this regard, a great advantage of RISs could lie in their low deployment and operation costs. Due to their mostly passive nature, they have low energy demands and unlike the deployment of additional base stations, they do not require a high capacity communication backhaul, but only some control link, which could be realized by in-band or out-of-band signaling. For this reason, the fixed installation of RISs on building surfaces, noise barriers, and other static surfaces in road traffic would lead to a SRE for several applications including but not limited to vehicular ones.

In addition, a dynamic and on-demand provisioning of RISs by mobile entities like UAVs is conceivable as proposed in $[2,19]$. The mobility in the three-dimensional space allows for a high flexibility and adaptability to varying requirements. For example, in case of a traffic accident, a UAV with a mounted RIS could increase the network capacity at the crash site. This may allow for forwarding comprehensive and detailed situation information and preparation of the rescue forces. In addition, such a UAV may supply a RISenhanced coverage to intercept network load peaks for a cluster of vehicles within a traffic congestion. Thus, dynamic cluster hovering constitutes another challenge of a dynamic coverage enhancement by means of dedicated RIS-UAVs. The dynamic placement of RISs may even accelerate the exploration of locations, which are best 


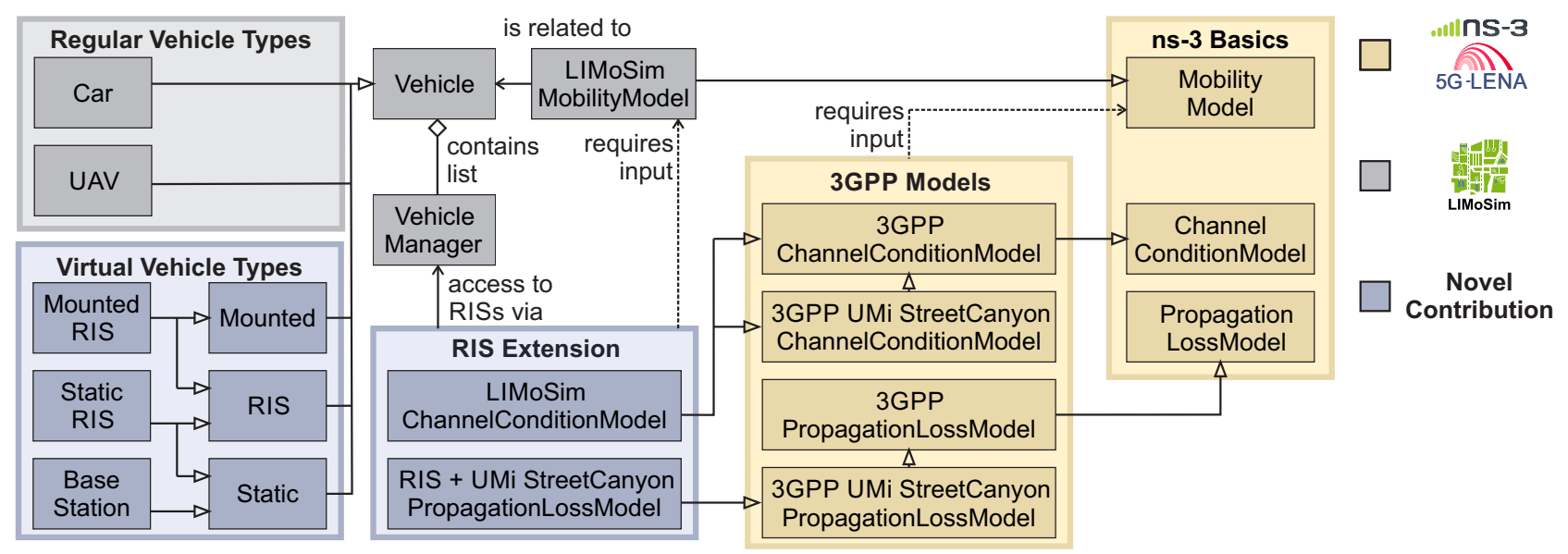

Figure 3: Details on the RIS model integration into and extension of our joint mobility and network simulation framework LIMoSim with ns-3/5G-LENA. On the basis of generic ns-3 classes, the 3GPP channel model and LIMoSim mobility framework are extended to account for RIS-enabled propagation paths in addition to the direct paths, which might be obstructed by buildings in some circumstances.

suited for a fixed and permanent deployment. In this way, machine learning is applicable not only to optimize the UAV trajectory, but also to derive recommendations for static RIS installations [10, 16].

Even cooperative mechanisms for proactive steering mmWave beams and their RIS-controlled reflections based on the predicted trajectories of the mobile vehicles constitute a promising field of research. Initial feasibility studies such as [8] could be carried on towards predictive steering algorithms, which among other objectives may take a reduction of handoffs between LOS and RIS reflection paths into account.

Future vehicular communication environments may integrate the RIS technology even deeper: As a vision, all kinds of vehicles could be coated with meta-material. Like the holographic MIMO surfaces in [6], this outer layer could be used for both an active communication of the vehicle itself and to support the SRE for wireless communications in its vicinity. This would lead to a vast amount of available RISs in urban and vehicular environments. Different from their discussed dedicated (static or dynamic) deployment with the primary purpose of enhancing wireless communications, a massive amount of RISs would enrich the SRE subsidiary. At the same time, the vehicles and objects with integrated RIS functionality pursue their usual transportation task and opportunistically offer a reflection occasion like the bus depicted at the bottom right of Figure 2.

In general, vehicular applications necessitate a timely control of the RIS configuration (particularly its reflection direction) due to the highly mobile nature and the resulting volatile radio channel. Their opportunistic utilization requires a distributed and predictive control and exploitation of the RIS resources. However, platoons of trucks and UAV swarms may profit form such a design, since they embody huge obstructions or lack multipath richness at the aerial wireless channel, respectively. Even innovative business models are imaginable, where surface resources could be offered to be leveraged on demand for intelligent reflections.

\section{SIMULATION ENABLED EVALUATION APPROACH}

As pointed out before, the wireless communications of ITS - especially in the mmWave and $\mathrm{THz}$ bands - may highly profit from smart radio environments. By means of joint mobility and network simulations, this section elaborates on the suggested opportunities of the RIS technology for hybrid vehicular communications.

\subsection{Simulation Framework}

The simulation environment is based on our LIMoSim framework from [14] with its system architecture model extended by RIS support as depicted in Figure 1.

In terms of vehicular mobility simulations, it implements UAV and motor vehicle models. The vehicles' environment includes a road topology, static obstacles like buildings, and a height profile of the terrain allowing for the application of geometry-based radio channel and signal propagation models. While the road topology and the buildings can be imported from OpenStreetMap, the terrain's height profile may be available depending on the region, e.g. through the pan-European digital surface model EU-DEM.

As a model for the communication network, the network simulator 3 (ns-3) and its 5G new radio (NR) module 5G-LENA [13] is coupled with the framework and extended by the RIS path loss model of [11] to account for RIS-enabled NLOS reflection paths. While this work focuses on the evaluation of RIS-enhanced network coverage, the integration of control links into the 5G NR signaling and the interaction with beam management procedures may be addressed in future work.

Finally, alongside a graphical visualization of the simulation scenario, the event-driven system simulation model offers performance indicators for a detailed evaluation of the simulation results. With this, the model facilitates the analysis of mobilityand topology-aware applications like predictive mmWave beam 
Calculate propagation loss between vehicle $A$ and vehicle $B$

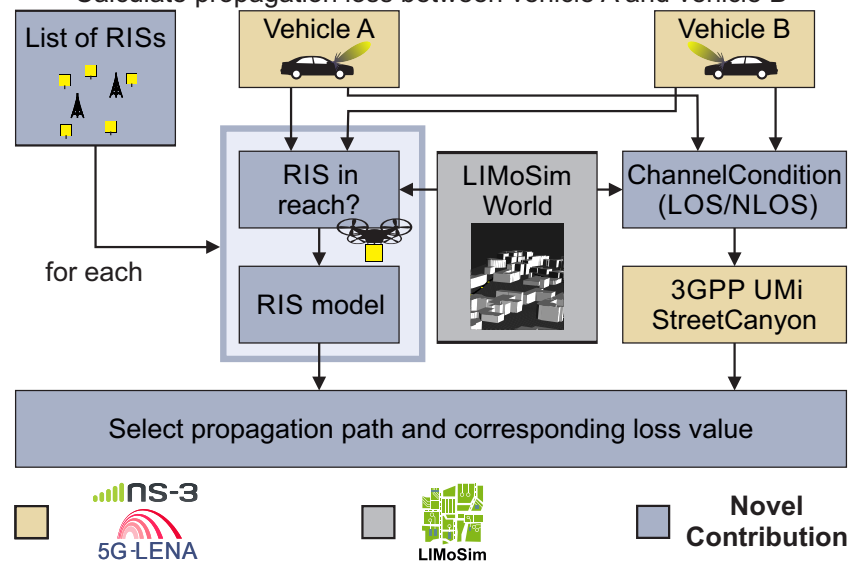

Figure 4: Process of propagation loss calculation within the simulation model. The channel condition (LOS or NLOS) as well as the RIS availability is determined by examination of the obstacles within the LIMoSim world. With a perfect knowledge of all available paths and their corresponding losses, the best-suited path can be selected to evaluate the general potential of the utilization of RIS reflected paths for coverage enhancements.

steering on the one hand. On the other hand, RIS network planning for the proposed dedicated or opportunistic utilization of both static and dynamic RISs can be applied also taking the surface dimensioning and its construction design into account.

Apart from this overview of the simulation model architecture, Figure 3 comprises more details on the integration of the RIS propagation loss model into LIMoSim: In ns-3, basic classes are provided to allow for modeling the mobility of nodes, channel conditions and the propagation loss of a wireless signal transmission. As one implementation, the 3rd generation partnership project (3GPP) channel models [1] are available therein, also forming the basis for $5 \mathrm{G}$ network simulations in the mmWave domain as elaborated in [13]. Especially, the 3GPP urban micro-cell (UMi) street canyon scenario is appropriate for simulations of the selected environment.

However, the associated channel condition model is derived from a probability distribution and needs to be replaced, since LIMoSim allows for a geometry-based assessment of the LOS condition. To retrieve the required geometry information like the vehicle locations, LIMoSim implements a derived mobility model, which is linked to its vehicle class.

For the introduction of RIS objects, further vehicle subclasses are generated. In doing so, RISs can be integrated as either static vehicles (i.e. they stay on a predefined position) or mounted onto some other vehicle (i.e. following its movement with a fixed offset). As a result, they are made available to the extended models for assessing the channel condition and calculating the propagation loss by means of the LIMoSim vehicle manager class, which holds a list of all vehicle instances defined in the simulation.

As further detailed in Figure 4, the RIS extension is thus able to screen the RISs in reach, i.e. those with LOS condition to both transmitter and receiver. To determine the availability of a certain
RIS in terms of existing LOS, obstacles like buildings are taken into account by means of the LIMoSim world singleton. This allows for the assessment of the channel conditions of both the direct path between transmitter and receiver as well as the reflection paths through a RIS from the list. As already mentioned, the vehicles' mobility models are available for geometric considerations like computing their horizontal or 3D distance as required by the propagation loss model. Beside the computation of the propagation loss of the direct path, which may be LOS or NLOS, the RIS path loss model from [11] is applied for each of RIS with LOS condition to both transmitter and receiver. The simulation solely takes first order reflections into account for the time being, as this is currently a constraint of the implemented model from [11]. Finally, within the simulation, all available RIS reflection paths can be evaluated and compared to the supposedly obstructed direct path.

\subsection{RIS Application Scenarios}

For the case study, a university campus is chosen as simulation area with some notional base station at a central location. As described in the previous section, the topology model includes buildings and roads from OpenStreetMap as well as a height profile to also account for the rather hilly terrain. Since the communication link performance highly depends on the LOS conditions, this realistic model of the distribution of obstacles allows for a geometry-based evaluation of the network coverage with regard to the aforementioned channel and propagation loss models.

As a first sample vehicular application, a campus shuttle bus trajectory covers the roads surrounding the campus area. The base station operates a $5 \mathrm{G}$ NR mobile network at the mmWave domain. Hence, the experienced path loss at the vehicle bears on the distance to the base station as well as on the LOS condition. However, due

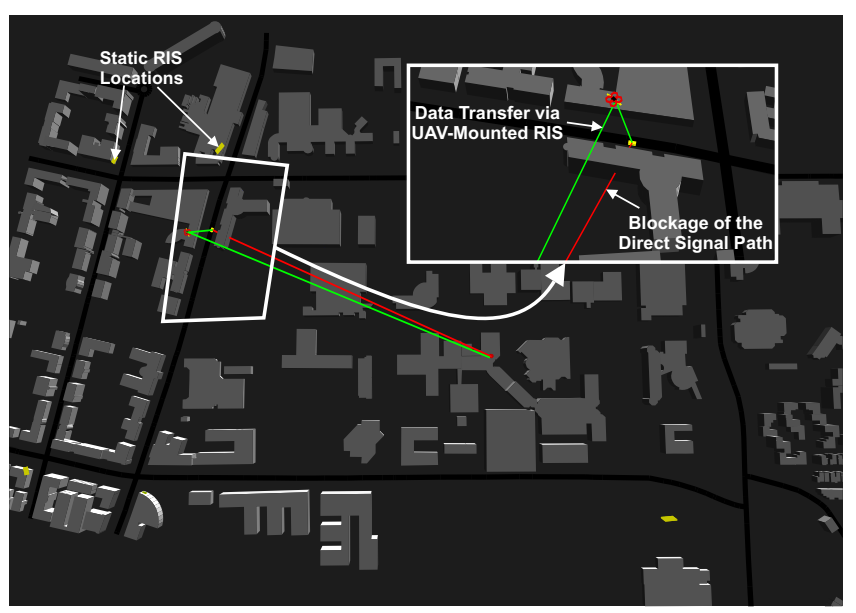

Figure 5: Excerpt of the simulation scenario (Map data: (1) OpenStreetMap Contributors, CC BY-SA). At the university campus, both the static deployment of RISs and a RIS mounted on a UAV improve the coverage of a notional, central located base station. Especially obstructed areas are supplied with a RIS-enhanced communication link mitigating the poor mmWave propagation at NLOS conditions. 
Table 1: Simulation details

\begin{tabular}{|c|c|}
\hline Objective & $\begin{array}{l}\text { Vehicular network coverage analysis with } \\
\text { static or UAV mounted RIS and central } \\
\text { base station }\end{array}$ \\
\hline Scenario & $\begin{array}{l}\text { University campus as depicted in Figure } 5 \text {, } \\
\text { a) Base station to vehicle } \\
\text { b) Vehicle to vehicle with } 400 \mathrm{~m} \text { initial dis- } \\
\text { tance }\end{array}$ \\
\hline $\begin{array}{l}\text { Propagation loss } \\
\text { model }\end{array}$ & $\begin{array}{l}\text { Direct path: 3GPP UMi Street Canyon ac- } \\
\text { cording to [1], } \\
\text { Reflection path: RIS model from [11] }\end{array}$ \\
\hline $\begin{array}{l}\text { Channel condition } \\
\text { model }\end{array}$ & $\begin{array}{l}\text { deterministic based on geometry (direct } \\
\text { path), considering buildings as obstacles }\end{array}$ \\
\hline Beam management & ideal, refer to $5 G-L E N A[13]$ \\
\hline RIS related & \\
\hline Shape/dimension & square, $0.5 \mathrm{~m} \cdot 0.5 \mathrm{~m}$, operated @28 GHz \\
\hline Deployment & $\begin{array}{l}\text { a) Static, for enhanced street canyon cov- } \\
\text { erage } \\
\text { b) Mounted on UAV, for dynamic supply }\end{array}$ \\
\hline Control & ideal/not considered \\
\hline RIS-UAV & $\begin{array}{l}\text { Follows the vehicle at a height of } 60 \mathrm{~m} \text {, ap- } \\
\text { plies fixed RIS downtilt of } 30^{\circ} \text {, horizontally } \\
\text { aligns RIS with } \\
\text { a) base station or b) second vehicle }\end{array}$ \\
\hline
\end{tabular}

to the numerous buildings, the LOS coverage is initially rather poor as evaluated in the next subsection.

Figure 5 portrays the campus setting from an aerial perspective highlighting in green color the currently utilized RIS reflection path to the vehicle due to an obstructed LOS (red line). In this scene, the RIS is carried by an UAV, but up to seven additional static RISs are deployed throughout the entire setting.

As a second vehicular use case, a V2V mmWave communication link is studied, where one vehicle tracks the other. Starting from different crossroads, their initial distance is about $400 \mathrm{~m}$ and may vary during the pursuit due to the mobility and acceleration model.

Such a mobile communication link could for example be leveraged to share sensor data for a collective perception of the vehicles' environment and to coordinate maneuvers in case of cooperative autonomous driving. While there is presumably a LOS situation when driving straight ahead, buildings at corners may lead to obstructions during turns degrading the link performance.

For this reason, the introduction of RISs may lead to a coverage enhancement especially at the NLOS regions. During preparatory intuitive and straightforward tests of different RIS locations, it turned out, that for the first case with a static base station, a RIS deployment near crossroads is effective and preferable to supply two road sections (street canyons) with an improved coverage by first order reflections via the RISs. A total of seven RISs is introduced into the setting to allow for a comprehensive improvement of the experienced path loss as evaluated in the subsequent section. Although the RIS deployment is conducted in an arbitrary manner, works like [10] propose sophisticated solutions for an automated placement.

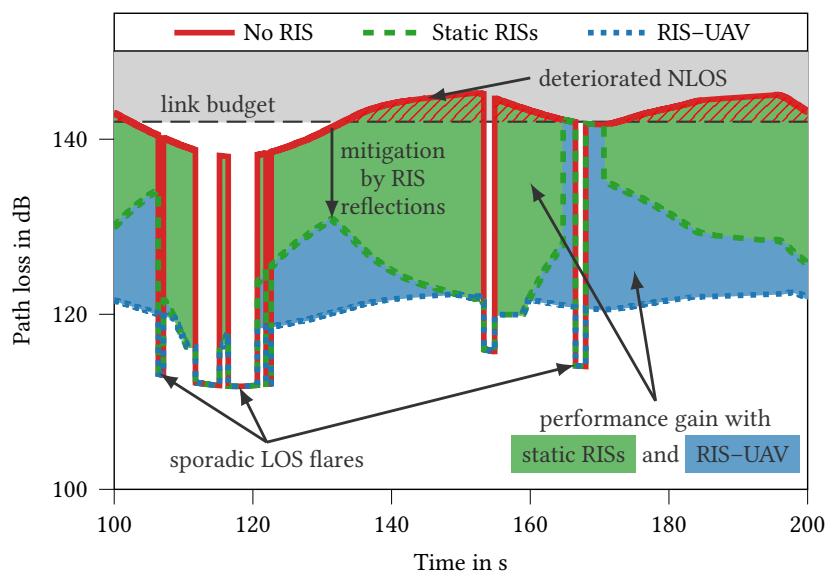

Figure 6: Exemplary excerpt of the path loss over time for the three cases: Without RIS, with deployed static RISs, and with additional support by a RIS mounted on a UAV. While the deteriorated NLOS link is sporadically improved by means of transient LOS flares, RIS-enabled reflection paths are able to mitigate the path loss despite the absence of a LOS condition. The colored areas measure the performance gain as product of mitigated path loss and time.

In contrast to a fixed placement of RISs for example as installation on building walls or at light poles, a dynamic and situational deployment may be feasible by mounting a RIS on a UAV leveraging its advantageous 3D movement abilities. To evaluate this approach, a RIS-UAV follows the designated vehicle at a fixed height of $60 \mathrm{~m}$ in the subsequent simulations. This RIS has a fixed downtilt of $30^{\circ}$ and aligns horizontally towards the base station by applying an angle of yaw to the sample quadrotor UAV accordingly. Beside this approach of tracking the designated vehicle while aligning the RIS towards the base station, a machine learning-based solution approach is presented in [19]. Table 1 summarizes the aforementioned details on the simulation model and its configuration.

\subsection{Performance Evaluation}

During the joint mobility and network simulation, the path loss is evaluated according to the LOS condition and current geometry between transmitter, RIS, and receiver. The underlying deterministic and geometry-based channel and propagation loss models thus provide new path loss values after a position update of any vehicle. Figure 6 depicts an excerpt of a time series of the experienced path loss for three different deployment strategies. Without any RIS (red line), the link performance is quite deteriorated at the predominant absence of LOS conditions. A LOS path is only sporadically available and rather transient due to the high blockage probability. However, the introduction of static RISs (dashed green line) mitigates the path loss by selecting a RIS reflection path in case of NLOS conditions. The additional utilization of a RIS mounted on a UAV mitigates the path loss even more significantly. It leads to a more consistent time response compared to the static RISs and drastically reduces the experienced path loss. With an assumed link budget of $142 \mathrm{~dB}$ according to [7], the RIS-enhanced coverage is 
able to avoid outages as subsequently analyzed in more detail. In addition, the green and blue areas illustrate the performance gain in terms of the product of path loss and time when utilizing static RISs and a RIS-UAV, respectively. This means, that the size of this area measures the advantage of the associated RIS deployment.

As the next step, the overall coverage of a RIS-enhanced base station is statistically analyzed in Figure 7. The empirical cumulative distribution function (ECDF) illustrates the statistical distribution of the experienced path loss levels for different RIS deployment strategies. Initially, the central base station placement appears questionable when not using any RIS, since it only covers $26 \%$ of the whole track. However, the successive introduction of statically deployed RISs improves the path loss to meet the link budget requirements. A comprehensive coverage is achievable by deploying seven static RISs. Nevertheless, the amount of added RISs can be balanced to cater for a distinct coverage level and path loss distribution. Finally, with additional support by a private RIS mounted on a UAV, the path loss is again significantly improved as also seen before. Albeit such a UAV can only supply a single vehicle or a cluster of vehicles within the same area. According to [11], the reduced path loss with the UAV-RIS is realizable due to a shorter distance to either the transmitter or receiver. In contrast to varying distances to the static RISs, the UAV is able to mostly keep the mounted RIS at a short distance.

In the $\mathrm{V} 2 \mathrm{~V}$ use case, the path loss is lower in general due to the shorter distance between the vehicles and higher LOS probability of them driving behind one another. In spite of that, utilizing static RISs at the turns and the UAV-RIS lead again to improved propagation conditions as evaluated in Figure 8. Since the predominant LOS condition leads to the same path loss measurements regardless of the RIS strategy as already observed in Figure 6, Figure 8

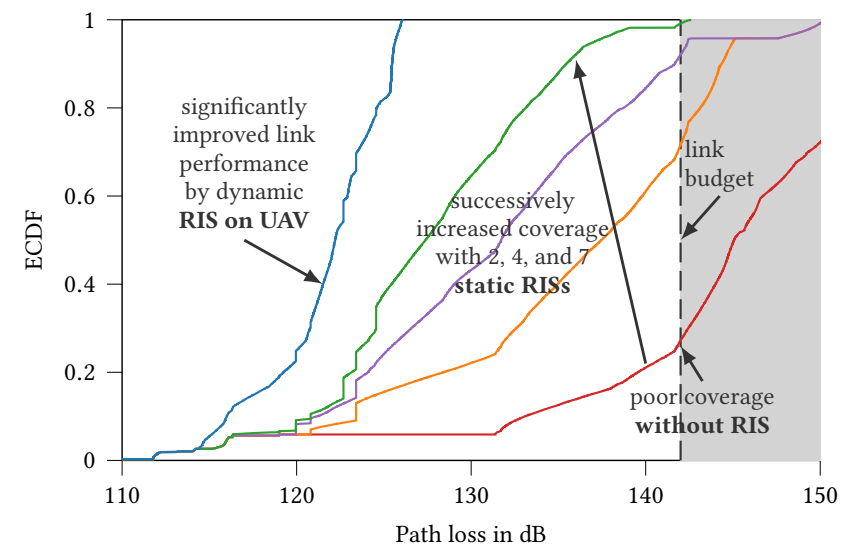

Figure 7: Statistical analysis of the overall coverage of a RISenhanced base station. While the link performance degrades without RIS, the successive introduction of static RISs enables meeting the link budget. The additional utilization of a RIS mounted on a UAV, which follows the vehicle, significantly improves the link reliability and grants path losses even below $127 \mathrm{~dB}$ in the underlying scenario.

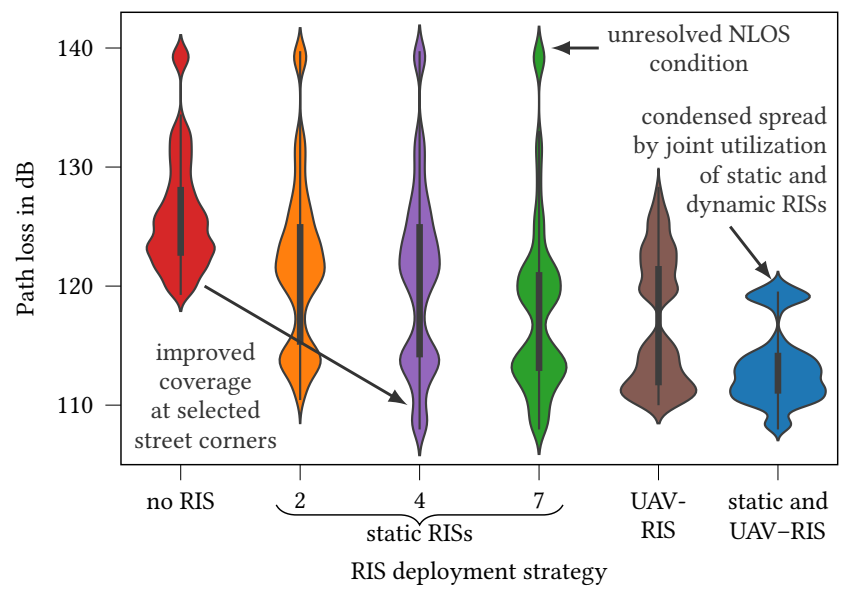

Figure 8: Statistical analysis of NLOS path loss of RISenhanced V2V communications. Due to the predominant LOS condition, this analysis focuses on the NLOS regions, which constitute about $15 \%$ of the evaluated trajectory. In general, the short distance between the vehicles lead to substantially lower path losses than in the base station scenario. However, the successive allocation of static RISs improve the experienced path loss during turns, where the LOS is obstructed by buildings at the street corner. Again, the combined utilization of both the static RISs and the dynamic RIS-UAV leads to notable improvements with a maximum path loss of $120 \mathrm{~dB}$.

focuses on the path loss under NLOS conditions, which are especially predominant at street corners. In general, the introduction of a RIS at a LOS obstructing street corner causes a mitigated path loss. However, a large number of RIS would be required to cover all occurring blockages during the track. In case of the deployment of only some static RISs, the path loss distribution partially still exceeds $140 \mathrm{~dB}$. This is not the case for the UAV-RIS. When utilizing both the static and the UAV-RIS, an overall reduced path loss with a condensed spread limited to a maximum of $120 \mathrm{~dB}$ can be seen as blue or rightmost violin of Figure 8 . This further reduction can again be explained by the distance dependency of the path loss model: While the UAV-RIS may have a low mean distance to the vehicle, the static RISs on buildings and light poles have a reduced altitude compared to the UAV and thus they may also have an even lower minimum distance when the vehicle is passing by.

In summary, the simulation results prove the advantageous coverage in terms of path loss when utilizing RISs. While a static deployment has only a limited range for coverage or path loss enhancement, the RIS-UAV is able to continuously supply reflection occasions dedicated to a single vehicle (or a local cluster of vehicles). Even V2V communications may profit from the RIS technology, since the path loss degrades under NLOS conditions, which are especially present at street corners. The combination of static and dynamic RISs turns out to join the advantages of both deployment strategies: On the one hand, a static RIS is dedicated to a certain street canyon or cross road and is thus able to drastically improve the experienced path loss but only at a limited area. On the other 
hand, the dynamic RIS, which is intentionally brought close to the vehicle by an UAV, can supply improved coverage anywhere but maybe with some restrictions due to no-fly zones or a constrained minimum altitude and its power consumption. As discussed earlier, future vehicular environments may even utilize reconfigurable intelligent surfaces in an opportunistic manner, whenever the direct paths degrades and a suited RIS mounted on a UAV or bus is intentionally or by chance in reach.

\section{CONCLUSION}

The RIS technology is believed to bear great potential for future wireless communications. Especially, these intelligent surfaces can be leveraged to facilitate the utilization of the volatile mmWave and $\mathrm{THz}$ bands within obstructed LOS conditions.

In this work, we introduced concepts for the application of RISs in vehicular environments, where not only a dedicated deployment of static or mobile RIS resources enriches the SRE, but also their opportunistic utilization when installed on any kind of building and vehicle surface, which is not (primarily) dedicated to enhance the network supply. The proposed system architecture model gives insights in applications like RIS network planning, while our sophisticated simulation framework has been extended to allow for a joint mobility and network based system-level evaluation of RIS reflections and SREs.

Results show, that static and dynamic deployments of RISs can successively eliminate dark zones and reduce the path loss allowing for the balance between the number of introduced RISs and the link performance.

The discussed opportunistic utilization and thereby required distributed control of the configured reflection direction through the network will be addressed in future work.

\section{ACKNOWLEDGMENTS}

Part of this work has been supported by Deutsche Forschungsgemeinschaft (DFG) within the Collaborative Research Center SFB 876 "Providing Information by Resource-Constrained Analysis", projects A4 and B4 as well as the German Federal Ministry of Education and Research (BMBF) for the project A-DRZ (Establishment of the German Rescue Robotics Center, 13N14857) and the Ministry of Economic Affairs, Innovation, Digitalization and Energy of the state of North Rhine-Westphalia in the course of the Competence Center 5G.NRW under grant number 005-01903-0047.

\section{REFERENCES}

[1] 3rd Generation Partnership Project (3GPP). 2020. Study on channel model for frequencies from 0.5 to $100 \mathrm{GHz}$. Technical Report. 3GPP TR 38.901, V16.1.0.

[2] Aly Sabri Abdalla, Talha Faizur Rahman, and Vuk Marojevic. 2020. UAVs with reconfigurable intelligent surfaces: Applications, challenges, and opportunities. arXiv preprint 2012.04775 (2020).

[3] Ian F. Akyildiz, Ahan Kak, and Shuai Nie. 2020. 6G and beyond: The future of wireless communications systems. IEEE Access 8 (2020), 133995-134030. https:
//doi.org/10.1109/ACCESS.2020.3010896

[4] Ahmed Al-Hilo, Moataz Samir, Mohamed Elhattab, Chadi Assi, and Sanaa Sharafeddine. 2021. Reconfigurable Intelligent Surface Enabled Vehicular Communication: Joint User Scheduling and Passive Beamforming. arXiv preprint 2101.12247 (2021).

[5] Karsten Heimann, Adrian Marsch, Benjamin Sliwa, and Christian Wietfeld. 2020. Reflecting Surfaces for Beyond Line-Of-Sight Coverage in Millimeter Wave Vehicular Networks. In 2020 IEEE Vehicular Networking Conference (VNC). IEEE. https://doi.org/10.1109/VNC51378.2020.9318411

[6] Chongwen Huang, Sha Hu, George C. Alexandropoulos, Alessio Zappone, Chau Yuen, Rui Zhang, Marco Di Renzo, and Merouane Debbah. 2020. Holographic MIMO surfaces for $6 \mathrm{G}$ wireless networks: Opportunities, challenges, and trends. IEEE Wireless Communications 27, 5 (2020), 118-125. https://doi.org/10.1109/ MWC.001.1900534

[7] Shajahan Kutty and Debarati Sen. 2016. Beamforming for Millimeter Wave Communications: An Inclusive Survey. IEEE Communications Surveys and Tutorials 18, 2 (2016), 949-973. https://doi.org/10.1109/COMST.2015.2504600

[8] I. Mavromatis, A. Tassi, R. J. Piechocki, and A. Nix. 2017. mmWave system for future ITS: A MAC-layer approach for V2X beam steering. In 2017 IEEE 86th Vehicular Technology Conference (VTC-Fall). https://doi.org/10.1109/VTCFall. 2017.8288267

[9] Hamid Menouar, Ismail Guvenc, Kemal Akkaya, A. Selcuk Uluagac, Abdullah Kadri, and Adem Tuncer. 2017. UAV-Enabled Intelligent Transportation Systems for the Smart City: Applications and Challenges. IEEE Communications Magazine 55, 3 (2017), 22-28. https://doi.org/10.1109/MCOM.2017.1600238CM

[10] Konstantinos Ntontin, Alexandros-Apostolos A. Boulogeorgos, Dimitrios Selimis, Fotis Lazarakis, Angeliki Alexiou, and Symeon Chatzinotas. 2020. Reconfigurable intelligent surface optimal placement in millimeter-wave networks. arXiv preprint 2011.09949 (2020).

[11] Özgecan Özdogan, Emil Björnson, and Erik G. Larsson. 2020. Intelligent Reflecting Surfaces: Physics, Propagation, and Pathloss Modeling. IEEE Wireless Communications Letters 9, 5 (May 2020), 581-585. https://doi.org/10.1109/LWC.2019.2960779 arXiv:1911.03359

[12] Manuel Patchou, Benjamin Sliwa, and Christian Wietfeld. 2021. Flying Robots for Safe and Efficient Parcel Delivery Within the COVID-19 Pandemic. In 2021 IEEE 15th International Systems Conference. Vancouver, Canada. arXiv:2101.07877 http://arxiv.org/abs/2101.07877

[13] Natale Patriciello, Sandra Lagen, Biljana Bojovic, and Lorenza Giupponi. 2019. An E2E simulator for 5G NR networks. Simulation Modelling Practice and Theory 96 (Nov. 2019). https://doi.org/10.1016/j.simpat.2019.101933

[14] Benjamin Sliwa, Manuel Patchou, Karsten Heimann, and Christian Wietfeld. 2020. Simulating hybrid aerial- and ground-based vehicular networks with ns-3 and LIMoSim. In Proceedings of the 2020 Workshop on Ns-3. Gaithersburg, Maryland, USA. https://doi.org/10.1145/3389400.3389407 arXiv:2003.09829

[15] Odysseas Tsilipakos, Anna C. Tasolamprou, Alexandros Pitilakis, Fu Liu, Xuchen Wang, Mohammad Sajjad Mirmoosa, Dimitrios C. Tzarouchis, Sergi Abadal, Hamidreza Taghvaee, Christos Liaskos, Ageliki Tsioliaridou, Julius Georgiou, Albert Cabellos-Aparicio, Eduard Alarcón, Sotiris Ioannidis, Andreas Pitsillides, Ian F. Akyildiz, Nikolaos V. Kantartzis, Eleftherios N. Economou, Costas M. Soukoulis, Maria Kafesaki, and Sergei Tretyakov. 2020. Toward Intelligent Metasurfaces: The Progress from Globally Tunable Metasurfaces to Software-Defined Metasurfaces with an Embedded Network of Controllers. Advanced Optical Materials 8, 17 (sep 2020), 2000783. https://doi.org/10.1002/adom.202000783 arXiv:https://onlinelibrary.wiley.com/doi/pdf/10.1002/adom.202000783

[16] F. Venturini, F. Mason, F. Pase, F. Chiariotti, A. Testolin, A. Zanella, and M. Zorzi. 2021. Distributed reinforcement learning for flexible and efficient UAV swarm control. IEEE Transactions on Cognitive Communications and Networking (2021). https://doi.org/10.1109/TCCN.2021.3063170

[17] Qingqing Wu and Rui Zhang. 2020. Towards Smart and Reconfigurable Environment: Intelligent Reflecting Surface Aided Wireless Network. IEEE Communications Magazine 58, 1 (2020), 106-112. https://doi.org/10.1109/MCOM.001.1900107

[18] Yong Zeng, Qingqing Wu, and Rui Zhang. 2019. Accessing from the Sky: A Tutorial on UAV Communications for 5G and beyond. Proc. IEEE 107, 12 (Dec. 2019), 2327-2375. https://doi.org/10.1109/JPROC.2019.2952892 arXiv:1903.05289

[19] Qianqian Zhang, Walid Saad, and Mehdi Bennis. 2019. Reflections in the Sky: Millimeter Wave Communication with UAV-Carried Intelligent Reflectors. In IEEE Global Communications Conference (GLOBECOM). https://doi.org/10.1109/ GLOBECOM38437.2019.9013626 